\title{
Analysis of Planetary Gear of Toyota Rush AT 2012
}

\author{
Adam Fathlevi ${ }^{1, a}$, Rudi Suhradi Rachmat ${ }^{2, b}$, Azhari Sastranegara ${ }^{3, c}$ \\ 1,2,3 Mechanical Engineering Department, President University, Indonesia. \\ JI. Ki Hajar Dewantara, Jababeka Education Center, Cikarang, Bekasi \\ aadamfathlevi@gmail.com, brudi.sr@president.ac.id, cazhari.sastranegara@president.ac.id
}

\begin{abstract}
Abstrak.
Gear merupakan salah satu bagian terpenting dari sistem transmisi tenaga pada sebuah kendaraan, dan seiring berjalannya waktu kemungkinan terjadi beberapa kegagalan pada roda gigi tersebut. Namun, tidak ada informasi yang diberikan oleh pabrikan mobil mengenai umur dan bahan dari roda gigi tersebut. Laporan ini menyajikan analisis planetary gear yang digunakan pada Toyota Rush AT tahun 2012 yang dibuat tahun 2012. Penelitian ini bertujuan untuk: mempelajari mekanisme planetary gear dan cara kerjanya; untuk menganalisis gaya dan tekanan yang bekerja pada roda gigi; dan untuk memilih material roda gigi yang sesuai yang dapat menahan beban yang terjadi pada roda gigi. Metodologi penelitian diawali dengan mencari dimensi roda gigi dan rasio roda gigi. Gaya yang bekerja pada roda gigi dihitung berdasarkan torsi dan kecepatan putaran yang diberikan dalam spesifikasi mobil. Kemudian dilakukan pemilihan material berdasarkan besarnya tegangan yang diterapkan pada gigi roda gigi yang dihitung menggunakan formulasi standar AGMA. Terakhir, prediksi umur kelelahan roda gigi dihitung berdasarkan tekanan sebelumnya yang bekerja pada roda gigi. Dari hasil perhitungan didapatkan baja AISI 1050 sebagai material roda gigi terpilih yang dapat menahan tegangan tekuk sebesar $476 \mathrm{MPa}$ dan dikategorikan sebagai siklus kelelahan rendah hingga tinggi.
\end{abstract}

Kata kunci: roda gigi planet, roda gigi cincin, perlengkapan matahari

\begin{abstract}
.
Gear is one of the most important part of power transmission system in a vehicle, and as the time goes by there possibly some failure happened on the gears. However, there is no information provided by car manufacturer regarding the life and the material of the gears. This report presents the analysis of the planetary gear used in Toyota Rush AT year made of 2012. The objectives of the research are: to study the planetary gear mechanism and how it works; to analyze the force and stress acting on the gears; and to select a suitable gear material that can withstand against the loads that happen on the gear. The research methodology is started with finding the gear dimension and gear ratio. The force acting on gear is calculated based on the torque and rotation speed provided in car specification. Then the selection material is carried out based on the amount of stress applied on the gear teeth that were calculated using AGMA standard formulation. Finally, the fatigue life prediction of the gears is calculated based on the previous stress acting on the gears. The calculation result suggested AISI 1050 steel as the selected gear material that can carry the bending stress of $476 \mathrm{MPa}$ and categorized as low to high fatigue cycle.
\end{abstract}

Keywords: planetary gear, ring gear, sun gear.

\section{Introduction}

\section{Background.}

There is a view kind of gears, one of them is planetary gear. This kind of gear has the first component, which is ring gear, Pinion gear, and sun gear. This kind of gear usually can be seen on automatic transmission car. With the gear design that always been attach to produce friction that is 
more minimum than the other design set gear conventional, so small there is less possibility of the gear to be worn out.

In this thesis researcher will be focusing on the gear that is on the gear planetary on the automatic transmission car. Basically, the manufacturer is not giving the detail information about the material that is used on the gear. Hopefully with this research people will be aware of the material that is used.

Problem Statement.

1. How the mechanism and how it work on planetary gear?

2. How the condition of the gear that is causes by force that's happen on planetary gear?

3. What kind of material that is right to be use on planetary gear?

Objective.

1. To study the planetary gear mechanism and how it works.

2. To analyze the force and bending stress acting on gear using AGMA standard equation.

3. To select the gear material that can withstand against bending stress that happen on the gear.

Limitations.

1. Support analysis is referenced from a guidebook. Reference book is "Fundamental of Element Machine" SI version third edition by Steven R.Schmid, Bernard J.Hamrock, Bo O.Jacobson and "Machine Elements in Mechanical Design" fourth edition by Robert L. This book contains tables of calculated results based on American Gears Manufactures Association (AGMA).

2. This research is only using the planetary gear of Toyota Rush 2012 AT car.

3. The gear that is use in this research is helical gear

4. How the vehicle works is not consider as reference.

5. Comparison of the material only for acknowledging the strength between material, not cunt as the cost if using material that is been decided as comparison.

\section{Literature Review}

\section{Gears.}

Gears are among the most important power transmission elements. A gear is a rotating machine element having cut teeth which mesh with another toothed part, usually having teeth of similar size and shape, in order to transmit power. Two or more gears working together are called a "transmission" (or gear set) and can produce "mechanical advantage" and thus may be considered a simple machine. The mechanical advantage is a measure of the force or torque amplification that is obtained using mechanical devices.

Types of gears: spur gear, helical gear, bevel gear, worm gear, and planetary gear.

Planetary gear.

1. Planetary trains include three main components: a sun gear, a planet carrier with one or more planet gears on it, and a ring gear.

2. Planetary trains have two or more degrees of freedom and thus have two or more inputs.

3. Planetary gear trains are commonly used for obtaining different gear ratios by fixing one of the elements (the sun, the ring, or the planet carrier) and using one of the remaining two elements as an input and the other as an output. 


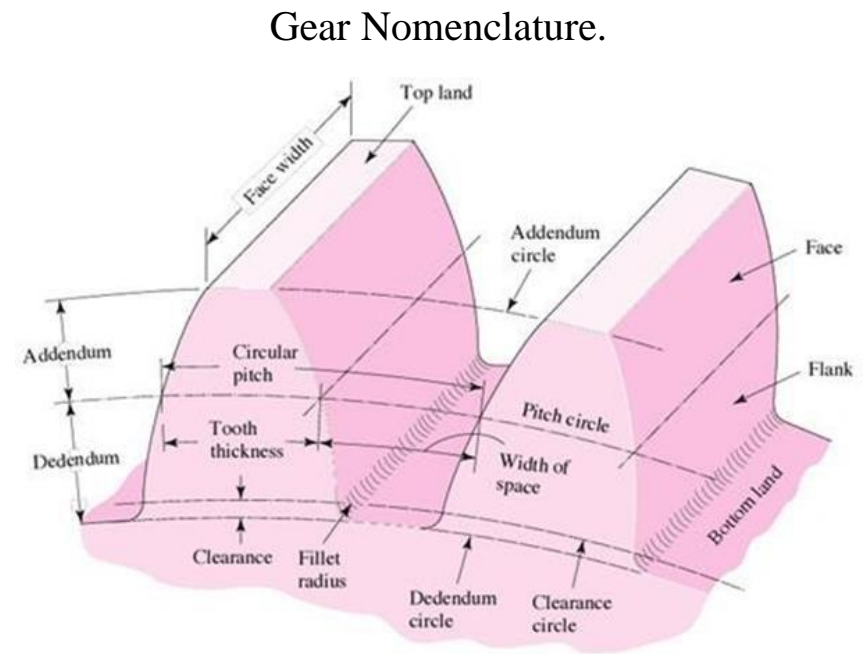

Spur Gears Teeth Profile.

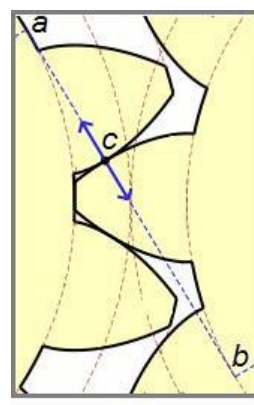

(1)

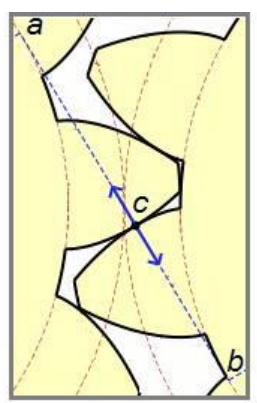

(2)

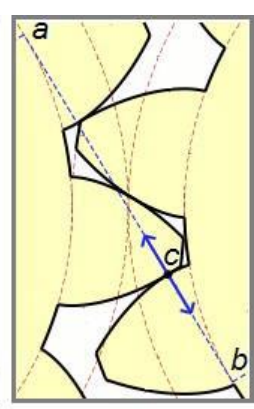

(3)

\section{Contact Ratio}

The contact ratio " $m c$ " defines the average number of teeth pairs in contact during meshing.

1. If $m c=1$ it means that only one pair of teeth is in contact at a time.

2. To reduce noise and possibility of impact it is recommended that $m c \geq 1.2$

3. The contact ratio increases with the number of teeth of a gear and for this reason, gears having less than ten teeth are not commonly used.

Helical Gear.

On helical gears, the teeth are inclined at an angle with the axis, that angle being called the heli.x angle. If the gear were very wide, it would appear that the teeth wind around the gear blank in a continuous, helical path. Helical gears has different pitches:

1. Circular pitch, p. Circular pitch is the distance from a point on one tooth to the corresponding point on the next adjacent tooth, measured at the pitch line in the transverse plane.

2. Normal circular pitch, $\mathrm{Pa}$. Normal circular pitch is the distance between corresponding points on adjacent teeth measured on the pitch surface in the normal direction.

3. Diametral pitch, Pd. Diametral pitch is the ratio of the number of teeth in the gear to the pitch diameter.

4. Normal diametral pitch, Pnd. Normal diametral pitch is the equivalent diametral pitch in the plane normal to the teeth

Forces, Torque and Power in Gearing.

$$
\text { Torque }=\frac{\text { Power }}{\text { Rotational Speed }}=\frac{P}{n}
$$




$$
\begin{gathered}
T=W_{t}(R)=W_{t}\left(\frac{D}{2}\right)=\frac{P}{n} \\
P=W_{t} \times v_{t}
\end{gathered}
$$

\section{Gear Quality.}

Factors of gear quality: index variation, toot alignment, tooth profile and root radius.

Stress in Gear Teeth.

1. Bending stress number

$$
\sigma_{b}=\frac{W_{t} P_{d} K_{o} K_{s} K_{m} K_{v} K_{b}}{b_{w} Y j}
$$

Where $\mathrm{W}_{\mathrm{t}}$ is tangential force, $\mathrm{P}_{\mathrm{d}}$ is diametral pitch of the tooth, $\mathrm{Y}_{\mathrm{j}}$ is geometric factor, $\mathrm{K}_{\mathrm{o}}$ is overload factor for bending strength, $\mathrm{K}_{\mathrm{s}}$ is size factor for bending strength, $\mathrm{K}_{\mathrm{m}}$ is load distribution factor for bending strength, $K_{v}$ is rim thickness factor and $K_{b}$ is dynamic factor for bending strength.

2. Overload factor, $\mathrm{K}_{\mathrm{o}}$

Suggested overload factors, $\mathrm{K}_{\mathrm{o}}$ :

\begin{tabular}{lcccc}
\hline & \multicolumn{4}{c}{ Driven Machine } \\
\cline { 2 - 5 } Power source & Uniform & $\begin{array}{l}\text { Light } \\
\text { shock }\end{array}$ & $\begin{array}{c}\text { Moderate } \\
\text { shock }\end{array}$ & $\begin{array}{r}\text { Heavy } \\
\text { shock }\end{array}$ \\
\hline Uniform & 1.00 & 1.25 & 1.50 & 1.75 \\
Light shock & 1.20 & 1.40 & 1.75 & 2.25 \\
Moderate shock & 1.30 & 1.70 & 2.00 & 2.75 \\
\hline
\end{tabular}

3. Size Factor, $\mathrm{K}_{\mathrm{s}}$

Suggested size factors, $\mathrm{K}_{\mathrm{s}}$

\begin{tabular}{lcc}
\hline $\begin{array}{c}\text { Diametral } \\
\text { pitch. } P_{J}\end{array}$ & $\begin{array}{c}\text { Metric } \\
\text { module, } m\end{array}$ & $\begin{array}{c}\text { Size factor. } \\
K\end{array}$ \\
\hline$\geq 5$ & 55 & 1.00 \\
4 & 6 & 1.05 \\
3 & 8 & 1.15 \\
2 & 12 & 1.25 \\
1.25 & 20 & 1.40 \\
\hline
\end{tabular}

4. Load Distribution Factor, $K_{m}$

Formula to calculate load distribution factor:

$$
K_{m}=1+C_{m c}\left(C_{p f} \times C_{p m}+C_{m a} \times C_{e}\right)
$$

Where: $\mathrm{C}_{\mathrm{mc}}$ is lead correction factor $\left(\mathrm{C}_{\mathrm{mc}}\right.$ is 1.0 for uncrowned teeth and 0.8 for crowned teeth), $\mathrm{C}_{\mathrm{pf}}$ is pinion proportion factor, $\mathrm{C}_{\mathrm{pm}}$ is pinion proportion modifier (see figure below), $\mathrm{C}_{\mathrm{ma}}$ is mesh alignment factor, and $\mathrm{C}_{\mathrm{e}}$ is mesh alignment correction factor. 


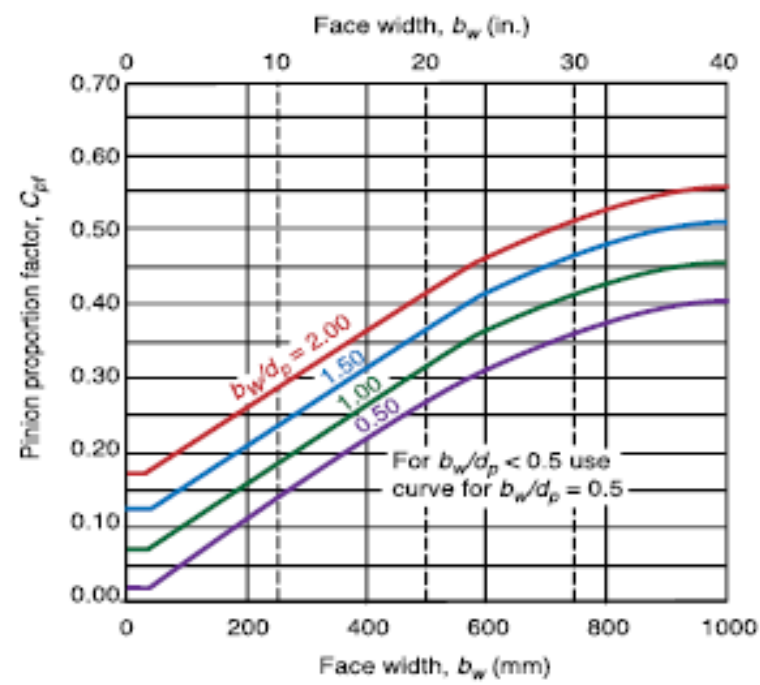

If $\mathrm{b}_{\mathrm{w}}<25 \mathrm{~mm}: C_{p f}=\frac{b_{w}}{10 d_{p}}-0.025$

For $25 \mathrm{~mm}<\mathrm{b}_{\mathrm{w}}<432 \mathrm{~mm}: C_{p f}=\frac{b_{w}}{10 d_{p}}-0.0375+0.000492 b_{w}$

For $432 \mathrm{~mm}<$ bw $\leq 1020 \mathrm{~mm}: C_{p f}=\frac{b_{w}}{10 d_{p}}-0.1109+0.000815 b_{w}-\left(3.53 \times 10^{-7}\right) b_{w}^{2}$

Pinion proportion modifier: $C_{p m}=1.0$ (for $S_{1} / S<0.175$ ) or 1.1 (for $S_{1} / S \geq 0.175$ ).

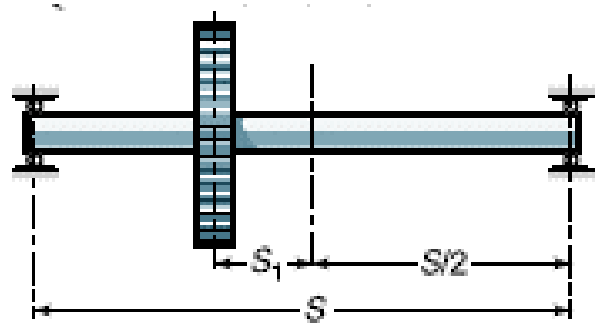

Mesh alignment correction factor: $\mathrm{C}_{\mathrm{e}}=0.80$ (when gearing is adjusted at assembly or when compatibility between gear teeth is improved by lapping) or 1.0 (for all other conditions).

Mesh alignment factor: $C_{m a}=A+B b_{w}+C b_{w}^{2}$

\begin{tabular}{lccc} 
If $b_{w}$ is in inches: & Table 2.7 Mesh alignment factor [35] \\
\hline Condition & $A$ & $B$ & $C$ \\
\hline Open gearing & 0.247 & 0.0167 & $-0.765 \times 10^{-4}$ \\
Commercial enclosed gears & 0.127 & 0.158 & $-1.095 \times 10^{-4}$ \\
Precision enclosed gears & 0.0675 & 0.0128 & $-0.926 \times 10^{-4}$ \\
Extraprecision enclosed gears & 0.000380 & 0.0102 & $-0.822 \times 10^{-4}$ \\
\hline If $b_{w}$ is in mm: & \multicolumn{4}{c}{} \\
\hline Condition & $A$ & $B$ & $C$ \\
\hline Open gearing & 0.247 & $6.57 \times 10^{-4}$ & $-1.186 \times 10^{-7}$ \\
Commercial enclosed gears & 0.127 & $6.22 \times 10^{-4}$ & $-1.69 \times 10^{-7}$ \\
Precision enclosed gears & 0.0675 & $5.04 \times 10^{-4}$ & $-1.44 \times 10^{-7}$ \\
Extraprecision enclosed gears & 0.000380 & $4.02 \times 10^{-4}$ & $-1.27 \times 10^{-7}$ \\
\hline
\end{tabular}

5. Dynamic Factor, Kv

Equation for dynamic factor:

Where $\mathrm{C}$ is 1 for $\mathrm{vt}$ in $\mathrm{ft} / \mathrm{min}, \mathrm{C}=\sqrt{ } 200=14.14$ for $\mathrm{vt}$ in $\mathrm{m} / \mathrm{s}$ and $\mathrm{A}=50+56(1.0-\mathrm{B})$. 


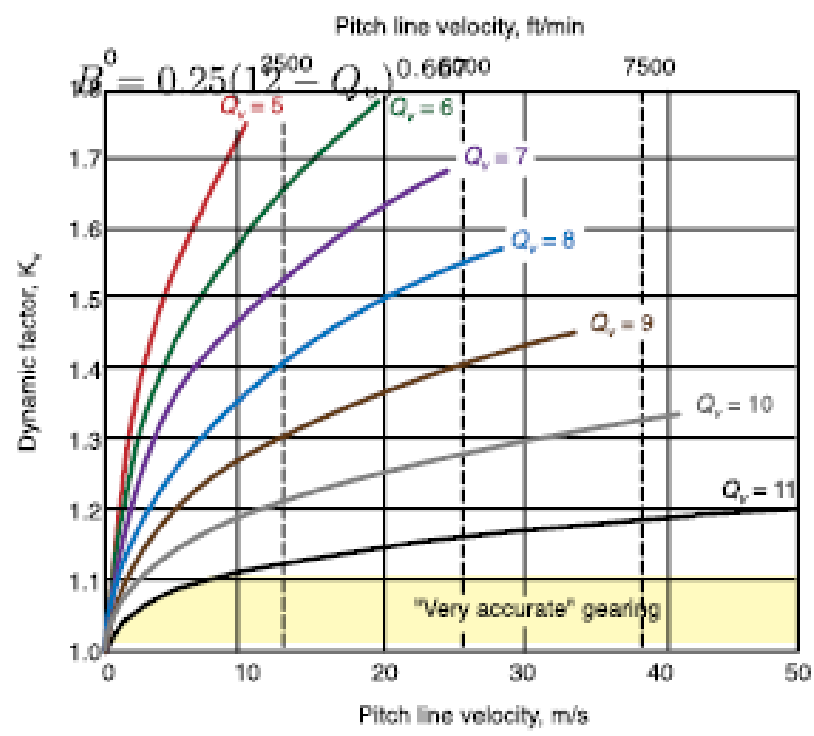

6. Rim Thickness Factor, $\mathrm{Kb}$

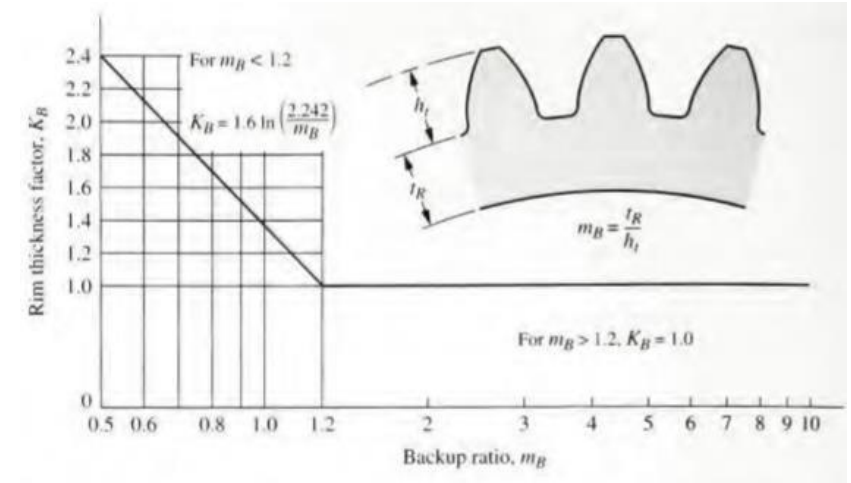

\section{Fatigue Life Cycle.}

Finite life. Low cycle fatigue $\left(10^{0}<\mathrm{N}<10^{3}\right)$ and high cycle fatigue $\left(\mathrm{N} \geq 10^{3}\right)$.

Infinite life $\left(\mathrm{N}>10^{6^{\sim} 7}\right)$..

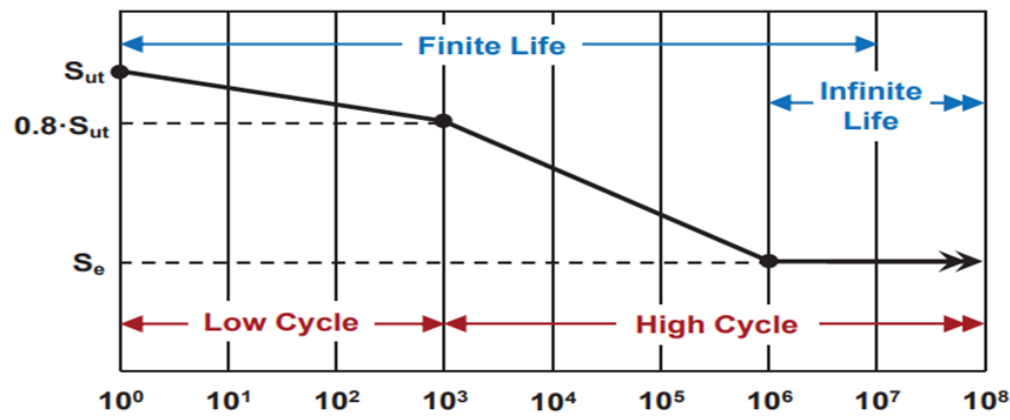

The correlation between $\mathrm{S} \& \mathrm{~N}$ in high cycle region can be obtained based on equation of the line:

$$
\begin{gathered}
B=\left(-\frac{1}{3}\right) \times \log \left(\frac{0.8 S_{u t}}{S_{e}}\right) \\
C=\log \left[\left(0.8 S_{u t}\right)^{2} / S_{e}\right. \\
N=10^{-\frac{c}{b}} \times S_{a}^{\frac{1}{b}}
\end{gathered}
$$




\section{Research Methodology}

\section{Research Framework.}

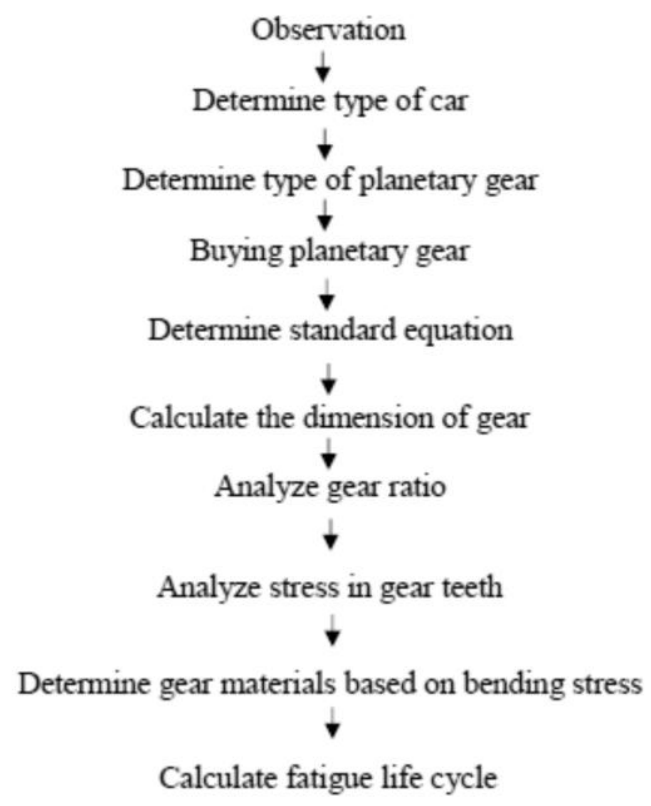

\section{Observation.}

Planetary gear to analyze from Toyota Rush AT 2012 with specification:

Power: $109 \mathrm{hp}=81215.9 \mathrm{~W}=81.2159 \mathrm{~kW}$

$\omega S U N: 6000 \mathrm{rpm}=628.3185 \mathrm{rad} / \mathrm{s}$

Torque: $129.2591 \mathrm{Nm}$

Module: $1.25 \mathrm{~mm}=0.00125 \mathrm{~m}$

Gear angle $\emptyset: 20^{\circ}=0.349$

Gear Dimension.

1. Sun Gear

$\mathrm{N}_{\text {SUN }}: 26$

Diametre (Dp): $\mathrm{N}_{\text {SUN }}$ x Module $=32.5 \mathrm{~mm}=0.0325 \mathrm{~m}$

Width (b): $22 \mathrm{~mm}=0.022 \mathrm{~m}$

Face Width (bw): b/cos $\emptyset=23.41 \mathrm{~mm}=0.02341 \mathrm{~m}$

Rim thickness (tr): 0.5

Teeth height (ht): 0.317

2. Planet 1 Gear

NPLANET: 20

Diametre (Dp): NPLANET X Module $=25 \mathrm{~mm}=0.025 \mathrm{~m}$

Width (b): $22 \mathrm{~mm}=0.022 \mathrm{~m}$

Face Width (bw): b/cos $\emptyset=23.41 \mathrm{~mm}=0.02341 \mathrm{~m}$

Rim thickness (tr): 0.5

Teeth height (ht): 0.317 
3. Planet 2 Gear

NPLANET: 20

Diametre (Dp): NPLANET X Module $=25 \mathrm{~mm}=0.025 \mathrm{~m}$

Width (b): $39 \mathrm{~mm}=0.039 \mathrm{~m}$

Face Width (bw): $\mathrm{b} / \cos \emptyset=41.5 \mathrm{~mm}=0.0415 \mathrm{~m}$

Rim thickness (tr): 0.5

Teeth height (ht): 0.317

4. Ring Gear

NPLANET: 71

Diametre (Dp): $\mathrm{N}_{\text {RING }}$ X Module $=88.75 \mathrm{~mm}=0.08875 \mathrm{~m}$

Width (b): $27 \mathrm{~mm}=0.027 \mathrm{~m}$

Face Width (bw): $\mathrm{b} / \cos \emptyset=28.73 . \mathrm{mm}=0.02873 \mathrm{~m}$

Rim thickness (tr): 0.5

Teeth height (ht): 0.317

\section{Gear Ratio.}

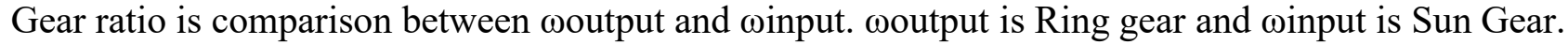

D RING: Nring x Module

$\mathrm{D}_{\text {SUN }}$ : Nsun x Module

DPLANET: Nplanet x Module

$\omega_{\text {SUN }}: 6000 \mathrm{rpm}=628.3185 \mathrm{rad} / \mathrm{s}$

Power: $109 \mathrm{hp}=81215.9 \mathrm{~W}=81.215 \mathrm{~kW}$

$$
\begin{gathered}
V_{\text {SUN }}=V_{P L A N E T}=V_{R I N G} \\
\omega_{R I N G}=\frac{V_{R I N G}}{R_{R I N G}} \\
\omega_{P L A N E T}=\frac{V_{P L A N E T}}{R_{P L A N E T}} \\
\text { Gear ratio }=\frac{\omega_{R I N G}}{\omega_{S U N}}
\end{gathered}
$$

Force Acting on Gear.

$$
\begin{gathered}
T_{S U N}=\frac{P}{\omega_{S U N}} \\
F_{S U N}=\frac{T_{S U N}}{R_{S U N}}=\frac{2 T_{S U N}}{D_{S U N}} \\
T_{R I N G}=\frac{P}{\omega_{R I N G}} \\
F_{R I N G}=\frac{T_{R I N G}}{R_{R I N G}}=\frac{2 T_{R I N G}}{D_{R I N G}} \\
T_{P L A N E T}=\frac{P}{\omega_{P L A N E T}}
\end{gathered}
$$




$$
F_{P L A N E T}=\frac{T_{P L A N E T}}{R_{P L A N E T}}=\frac{2 T_{P L A N E T}}{D_{P L A N E T}}
$$

\section{Analyzing of Planetary Gear.}

1. Sun Gear

Bending stress equation:

$$
\sigma_{b}=\frac{W_{t} P_{d} K_{o} K_{s} K_{m} K_{v} K_{b}}{b_{w} Y_{j}}
$$

Given: $\mathrm{Pd}$ is $800 \mathrm{~m}^{-1}$, dp is $32.5 \mathrm{~mm}, \mathrm{~b}$ is $22 \mathrm{~mm}, \mathrm{Y}_{\mathrm{j}}$ is $0.35, \mathrm{~K}_{\mathrm{o}}$ is 2 (moderate shock), $\mathrm{K}_{\mathrm{s}}$ is 1 (recommended values of size factor if module $<5 \mathrm{~mm}$ ), $\mathrm{C}_{\mathrm{mc}}$ is 1 (for uncrowned teeth), $\mathrm{C}_{\mathrm{pm}}$ is 1 (recommended values if $\left.\left(\mathrm{S}_{1} / \mathrm{S}\right)<0.175\right), \mathrm{C}_{\mathrm{e}}$ is 1 (for all other conditions), $\mathrm{t}_{\mathrm{R}}$ is 0,5 , and $\mathrm{h}_{\mathrm{t}}$ is 0.317 .

Solution:

Transmitted load:

$$
\begin{aligned}
& W_{t}=\frac{T}{\frac{1}{2} d p} \\
& b w=\frac{b}{\sin \theta}
\end{aligned}
$$

Load distribution factor:

$$
K_{m}=1+C_{m c}\left(C_{p f} \times C_{p m}+C_{m a} \times C_{e}\right)
$$

Find out $\mathrm{C}_{\mathrm{pf}}$ and $\mathrm{C}_{\mathrm{ma}}$ :

$$
\begin{aligned}
C_{p f} & =\frac{b w}{10 \times d p}-0.025 \\
C_{m a} & =A+B b w+C b w^{2}
\end{aligned}
$$

Thus

$$
C_{m a}=0.0675+5.04 \times 10^{-4} \times 0.023412 \pm 1.44 \times 10^{-7} \times 0.023412^{2}
$$

Dynamic factor:

$$
K_{v}=\left(\frac{A+C \sqrt{v t}}{A}\right)^{B}
$$

From AGMA bending stress equation table:

$$
\begin{gathered}
A=50+56(1-B) \\
B=0.25\left(12-Q_{v}\right)^{0.667} \\
C=14.14 \text { for vt in } \frac{m}{s}
\end{gathered}
$$

$\mathrm{Q}_{\mathrm{v}}$ is 11 (for automotive transmission) 
Rim thickness factor:

$$
\begin{gathered}
K_{b}=-2 m_{b}+3.4 \\
m_{b}=\frac{t_{R}}{h_{t}}
\end{gathered}
$$

2. Planet 1 Gear

AGMA bending stress equation:

$$
\sigma_{b}=\frac{W_{t} P_{d} K_{o} K_{s} K_{m} K_{v} K_{b}}{b_{w} Y_{j}}
$$

Given: $\mathrm{Pd}$ is $800 \mathrm{~m}^{-1}$, dp is $25 \mathrm{~mm}, \mathrm{~b}$ is $22 \mathrm{~mm}, \mathrm{Y}_{\mathrm{j}}$ is $0.35, \mathrm{~K}_{\mathrm{o}}$ is 1 (power source: uniform), $\mathrm{K}_{\mathrm{s}}$ is 1 (recommended values of size factor if module $<5 \mathrm{~mm}$ ), $\mathrm{C}_{\mathrm{mc}}$ is 1 (for uncrowned teeth), $\mathrm{C}_{\mathrm{pm}}$ is 1 (recommended values if $\left.\left(\mathrm{S}_{1} / \mathrm{S}\right)<0.175\right), \mathrm{C}_{\mathrm{e}}$ is 1 (for all other conditions), $\mathrm{t}_{\mathrm{R}}$ is $0,5, \mathrm{~h}_{\mathrm{t}}$ is 0.317 , and $\mathrm{K}_{\mathrm{b}}$ is 1 (conventional gear).

Solution:

$$
\begin{aligned}
& W_{t}=\frac{T}{\frac{1}{2} d p} \\
& b w=\frac{b}{\sin \theta}
\end{aligned}
$$

Load distribution factor:

$$
K_{m}=1+C_{m c}\left(C_{p f} \times C_{p m}+C_{m a} \times C_{e}\right)
$$

Find out $\mathrm{C}_{\mathrm{pf}}$ and $\mathrm{C}_{\mathrm{ma}}$ :

$$
\begin{aligned}
C_{p f} & =\frac{b w}{10 \times d p}-0.025 \\
C_{m a} & =A+B b w+C b w^{2}
\end{aligned}
$$

Dynamic factor:

$$
K_{v}=\left(\frac{A+C \sqrt{v t}}{A}\right)^{B}
$$

From AGMA bending stress equation table:

$$
\begin{gathered}
A=50+56(1-B) \\
B=0.25\left(12-Q_{v}\right)^{0.667} \\
C=14.14 \text { for vt in } \frac{m}{s}
\end{gathered}
$$

$\mathrm{Q}_{\mathrm{v}}$ is 11 (for automotive transmission)

3. Planet 2 Gear

AGMA bending stress equation:

$$
\sigma_{b}=\frac{W_{t} P_{d} K_{o} K_{s} K_{m} K_{v} K_{b}}{b_{w} Y_{j}}
$$

Given: $\mathrm{Pd}$ is $800 \mathrm{~m}^{-1}$, dp is $25 \mathrm{~mm}, \mathrm{~b}$ is $39 \mathrm{~mm}, \mathrm{Y}_{\mathrm{j}}$ is $0.35, \mathrm{~K}_{\mathrm{o}}$ is 1 (power source: uniform), $\mathrm{K}_{\mathrm{s}}$ is 1 (recommended values of size factor if module $<5 \mathrm{~mm}$ ), $\mathrm{C}_{\mathrm{mc}}$ is 1 (for uncrowned teeth), $\mathrm{C}_{\mathrm{pm}}$ 
is 1 (recommended values if $\left.\left(\mathrm{S}_{1} / \mathrm{S}\right)<0.175\right), \mathrm{C}_{\mathrm{e}}$ is 1 (for all other conditions), $\mathrm{t}_{\mathrm{R}}$ is $0,5, \mathrm{~h}_{\mathrm{t}}$ is 0.317 , and $\mathrm{K}_{\mathrm{b}}$ is 1 (conventional gear).

Solution:

$$
\begin{aligned}
& W_{t}=\frac{T}{\frac{1}{2} d p} \\
& b w=\frac{b}{\sin \theta}
\end{aligned}
$$

Load distribution factor:

$$
K_{m}=1+C_{m c}\left(C_{p f} \times C_{p m}+C_{m a} \times C_{e}\right)
$$

Find out $\mathrm{C}_{\mathrm{pf}}$ and $\mathrm{C}_{\mathrm{ma}}$ :

$$
\begin{aligned}
C_{p f} & =\frac{b w}{10 \times d p}-0.025 \\
C_{m a} & =A+B b w+C b w^{2}
\end{aligned}
$$

Dynamic factor:

$$
K_{v}=\left(\frac{A+C \sqrt{v t}}{A}\right)^{B}
$$

From AGMA bending stress equation table:

$$
\begin{gathered}
A=50+56(1-B) \\
B=0.25\left(12-Q_{v}\right)^{0.667} \\
C=14.14 \text { for vt in } \frac{m}{s}
\end{gathered}
$$

$\mathrm{Q}_{\mathrm{v}}$ is 11 (for automotive transmission)

4. Ring Gear

AGMA bending stress equation:

$$
\sigma_{b}=\frac{W_{t} P_{d} K_{o} K_{s} K_{m} K_{v} K_{b}}{b_{w} Y_{j}}
$$

Given: $\mathrm{Pd}$ is $800 \mathrm{~m}^{-1}$, dp is $88.75 \mathrm{~mm}, \mathrm{~b}$ is $27 \mathrm{~mm}, \mathrm{Y}_{\mathrm{j}}$ is $0.35, \mathrm{~K}_{\mathrm{o}}$ is 1 (power source: uniform), $\mathrm{K}_{\mathrm{s}}$ is 1 (recommended values of size factor if module $<5 \mathrm{~mm}$ ), $\mathrm{C}_{\mathrm{mc}}$ is 1 (for uncrowned teeth), $\mathrm{C}_{\mathrm{pm}}$ is 1 (recommended values if $\left(\mathrm{S}_{1} / \mathrm{S}\right)<0.175$ ), $\mathrm{C}_{\mathrm{e}}$ is 1 (for all other conditions), $\mathrm{t}_{\mathrm{R}}$ is $0,5, \mathrm{~h}_{\mathrm{t}}$ is 0.317 , and $\mathrm{K}_{\mathrm{b}}$ is 1 (conventional gear).

Solution:

$$
\begin{aligned}
& W_{t}=\frac{T}{\frac{1}{2} d p} \\
& b w=\frac{b}{\sin \theta}
\end{aligned}
$$


Load distribution factor:

$$
K_{m}=1+C_{m c}\left(C_{p f} \times C_{p m}+C_{m a} \times C_{e}\right)
$$

Find out $\mathrm{C}_{\mathrm{pf}}$ and $\mathrm{C}_{\mathrm{ma}}$ :

$$
\begin{aligned}
C_{p f} & =\frac{b w}{10 \times d p}-0.025 \\
C_{m a} & =A+B b w+C b w^{2}
\end{aligned}
$$

Dynamic factor:

$$
K_{v}=\left(\frac{A+C \sqrt{v t}}{A}\right)^{B}
$$

From AGMA bending stress equation table:

$$
\begin{gathered}
A=50+56(1-B) \\
B=0.25\left(12-Q_{v}\right)^{0.667} \\
C=14.14 \text { for vt in } \frac{m}{s}
\end{gathered}
$$

$\mathrm{Q}_{\mathrm{v}}$ is 11 (for automotive transmission)

Selecting Gear Material.

Thus the selection of material based on these data was chosen is AISI 1050 with properties:

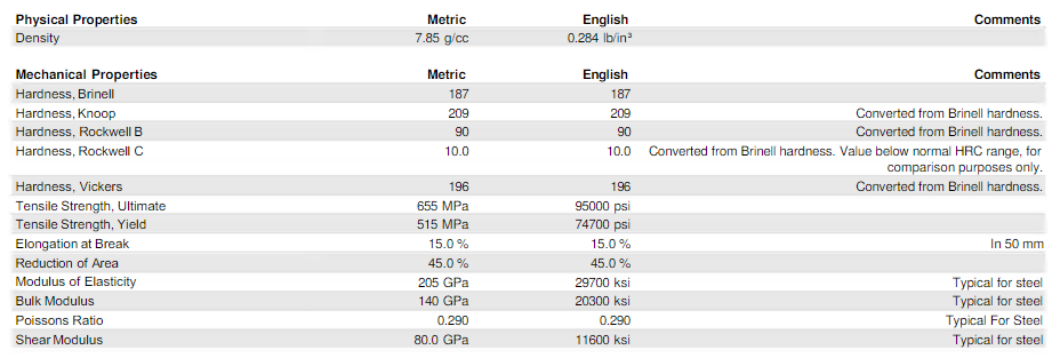

Selecting material is not consider cost material and cost manufacture.

Fatigue Life Cycle.

Given:

T: $129.2591 \mathrm{Nm}$

$D_{\text {spring: }} 0.08875 \mathrm{~m}$

$\mathrm{S}_{\mathrm{ut}}: 655 \mathrm{MPa}$

$\mathrm{S}_{\mathrm{e}}: 515 \mathrm{MPa}$

$$
\begin{gathered}
\text { Addendum }=1 \times \text { pitch } \\
\text { Dedendum }=1.25 \times \text { pitch }
\end{gathered}
$$




$$
\begin{gathered}
R_{d}=\frac{D_{p}}{2}+\text { addendum } \\
R_{a}=\frac{D_{p}}{2}-\text { dedendum } \\
w t_{\text {min }}=\frac{T}{R_{d}} \\
w t_{\text {max }}=\frac{T}{R_{a}}
\end{gathered}
$$

Calculation for $\sigma_{\mathrm{bmin}}$ and $\sigma_{\mathrm{bmax}}$ in below:

$$
\begin{aligned}
\sigma_{\text {bmin }} & =\frac{w t_{\min } P_{d} K_{o} K_{s} K_{m} K_{v} K_{b}}{b_{w} Y_{j}} \\
\sigma_{\text {bmax }} & =\frac{w t_{\max } P_{d} K_{o} K_{s} K_{m} K_{v} K_{b}}{b_{w} Y_{j}}
\end{aligned}
$$

Value for $\mathrm{Pd}, \mathrm{Ko}, \mathrm{Ks}, \mathrm{Km}, \mathrm{Kv}, \mathrm{Kb}$, bw and $\mathrm{Yj}$ is same as on chapter 3.1.5. The value from ring gear.

$$
\begin{gathered}
\text { Stress Amplitude } S_{a}=\frac{\sigma_{b \max }-\sigma_{b \min }}{2} \\
\text { Mean Stress } S_{m}=\frac{\sigma_{b \max }+\sigma_{b \min }}{2}
\end{gathered}
$$

Cycle Curve:

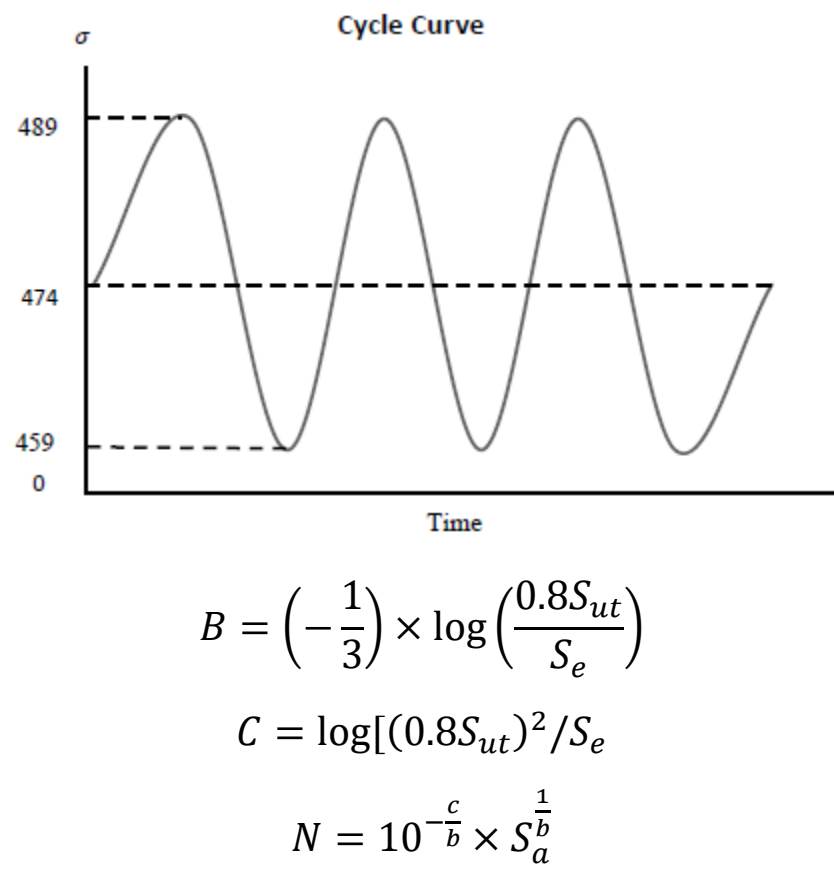


S-N Curve:

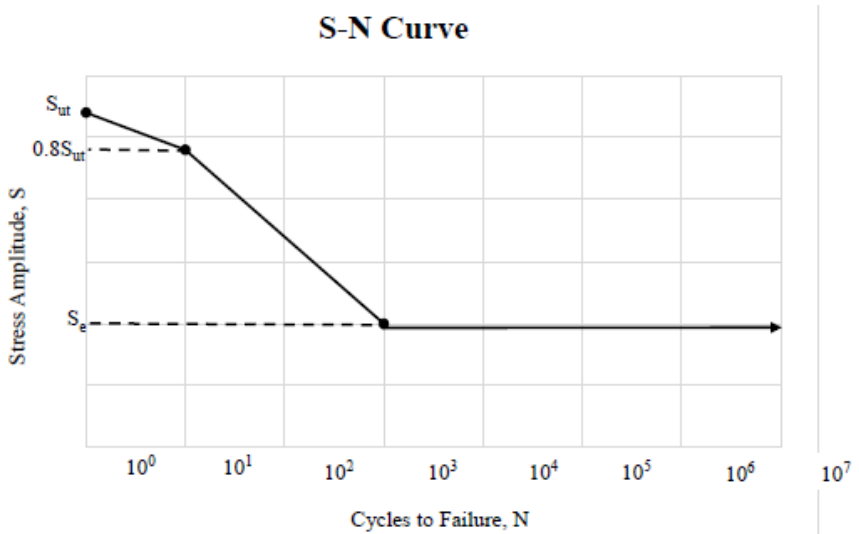

Based on results of existing calculation, the fatigue life cycle is categorized as low to high cycle. $\left(\mathrm{N} \approx 10^{3}\right)$.

\section{Discussion and Assessment Result}

Data Collection.

\begin{tabular}{|l|l|l|l|}
\hline No. & Description & Result & Unit \\
\hline 1 & Power $(\mathrm{P})$ & 81215.9 & $\mathrm{~W}$ \\
\hline 2 & Omega $(\omega)$ & 638.3185 & $\mathrm{rad} / \mathrm{s}$ \\
\hline 3 & Torque $(\mathrm{T})$ & 129.2591 & $\mathrm{Nm}$ \\
\hline 4 & Module $(\mathrm{m})$ & 0.00125 & $\mathrm{~m}$ \\
\hline 5 & Gear angle $(\phi)$ & 20 & $\circ$ \\
\hline 6 & Velocity $(\mathrm{v})$ & 10.21 & $\mathrm{~m} / \mathrm{s}$ \\
\hline 7 & $\begin{array}{l}\text { Geometry factor } \\
\left(\mathrm{Y}_{\mathrm{j}}\right)\end{array}$ & 0.35 & \\
\hline 8 & Diameter pitch $\left(\mathrm{P}_{\mathrm{d}}\right)$ & 800 & $\mathrm{~m}^{-1}$ \\
\hline
\end{tabular}

Gear Ratio.

Gear ratio result.

\begin{tabular}{|c|l|c|c|c|}
\hline No. & Description & Result & Unit & Ratio \\
\hline 1 & Ring gear & 88.75 & $\mathrm{~mm}$ & 3.55 \\
\hline 2 & Sun gear & 32.5 & $\mathrm{~mm}$ & 1.3 \\
\hline 3 & Planet gear & 25 & $\mathrm{~mm}$ & 1 \\
\hline
\end{tabular}

$\omega$ result.

\begin{tabular}{|c|l|c|c|}
\hline No. & \multicolumn{1}{|c|}{ Description } & Result & Unit \\
\hline 1 & $\omega_{\text {RING }}$ & 230 & $\mathrm{rad} / \mathrm{s}$ \\
\hline 2 & $\omega_{\text {SUN }}$ & 628.3185 & $\mathrm{rad} / \mathrm{s}$ \\
\hline 3 & $\omega_{\text {PLANET }}$ & 816.8 & $\mathrm{rad} / \mathrm{s}$ \\
\hline
\end{tabular}

For meshing gears the rotational motion and the power must be transmitted from the driving gear to the driven gear with a smooth and uniform positive motion and with minor frictional power loss. The fundamental law of gearing states that the common normal to the tooth profile at the point of contact must always pass through a fixed point, called the pitch point, in order to maintain a constant velocity 
ratio of the two meshing gear teeth. Since the velocity at this point must be the same for Contact Ratio and Gear Velocity.

$$
\text { Gear ratio }=\frac{\omega_{R I N G}}{\omega_{S U N}}=\frac{230 \mathrm{rad} / \mathrm{s}}{628.3185 \mathrm{rad} / \mathrm{s}}=0.366
$$

Force and Torque on Gear.

Torque result.

\begin{tabular}{|c|l|c|c|}
\hline No. & \multicolumn{1}{|c|}{ Description } & Result & Unit \\
\hline 1 & T $_{\text {SUN }}$ & 129.2591 & $\mathrm{Nm}$ \\
\hline 2 & $\mathrm{~T}_{\text {RING }}$ & 353.1126 & $\mathrm{Nm}$ \\
\hline 3 & T $_{\text {PLANET }}$ & 99.4318 & $\mathrm{Nm}$ \\
\hline
\end{tabular}

Force result.

\begin{tabular}{|c|l|c|c|}
\hline No. & \multicolumn{1}{|c|}{ Description } & Result & Unit \\
\hline 1 & F $_{\text {SUN }}$ & 7.954 & $\mathrm{kN}$ \\
\hline 2 & F $_{\text {RING }}$ & 7.957 & $\mathrm{kN}$ \\
\hline 3 & F & 7.954 & $\mathrm{kN}$ \\
\hline
\end{tabular}

Based on this result counted in gear ratio, got the torque in every gear. Within like that can be known Tring > Tsun > Tplanet. And the result in counted force in every gear can be get almost the same result.

Planetary Gear Calculation Result.

\begin{tabular}{|c|l|c|c|c|c|c|c|c|c|c|}
\hline No. & Description & $\mathrm{D}_{\mathrm{p}}$ & Unit & $\mathrm{N}$ & $\mathrm{W}_{\mathrm{t}}$ & Unit & $\mathrm{B}$ & Unit & $\mathrm{b}_{\mathrm{w}}$ & Unit \\
\hline 1 & Sun gear & 32.5 & $\mathrm{~mm}$ & 26 & 7954.407 & $\mathrm{~N}$ & 22 & $\mathrm{~mm}$ & 23.41 & $\mathrm{~mm}$ \\
\hline 2 & Planet 1 gear & 25 & $\mathrm{~mm}$ & 20 & 10424.12 & $\mathrm{~N}$ & 22 & $\mathrm{~mm}$ & 23.41 & $\mathrm{~mm}$ \\
\hline 3 & Planet 2 gear & 25 & $\mathrm{~mm}$ & 20 & 10424.12 & $\mathrm{~N}$ & 39 & $\mathrm{~mm}$ & 41.5 & $\mathrm{~mm}$ \\
\hline 4 & Ring gear & 88.75 & $\mathrm{~mm}$ & 71 & 2912.88 & $\mathrm{~N}$ & 27 & $\mathrm{~mm}$ & 28.73 & $\mathrm{~mm}$ \\
\hline
\end{tabular}

Planetary gear data factor result

\begin{tabular}{|c|l|c|c|c|c|c|}
\hline No. & Description & $\mathrm{K}_{\mathrm{o}}$ & $\mathrm{K}_{\mathrm{s}}$ & $\mathrm{K}_{\mathrm{m}}$ & $\mathrm{K}_{\mathrm{v}}$ & $\mathrm{K}_{\mathrm{b}}$ \\
\hline 1 & Sun gear & 2 & 1 & 1.11 & 1.1 & 0.245 \\
\hline 2 & $\begin{array}{l}\text { Planet 1 } \\
\text { gear }\end{array}$ & 1 & 1 & 1.36 & 1.1 & 1 \\
\hline 3 & $\begin{array}{l}\text { Planet 2 } \\
\text { gear }\end{array}$ & 1 & 1 & 1.19 & 1.1 & 1 \\
\hline 4 & Ring gear & 1.75 & 1 & 1.06 & 1.1 & 1 \\
\hline
\end{tabular}

Stress and transmitted load result for material recommendation:

\begin{tabular}{|c|l|c|c|}
\hline No. & \multicolumn{1}{|c|}{ Description } & Result & Unit \\
\hline 1 & $\sigma_{\mathrm{b}}$ & 476 & $\mathrm{MPa}$ \\
\hline 2 & $\sigma_{\max }$ & 489.8 & $\mathrm{MPa}$ \\
\hline 3 & $\sigma_{\min }$ & 459.8 & $\mathrm{MPa}$ \\
\hline 4 & $\mathrm{wt}_{\max }$ & 2997.3 & $\mathrm{~N}$ \\
\hline 5 & $\mathrm{wt}_{\min }$ & 2813.8 & $\mathrm{~N}$ \\
\hline
\end{tabular}


Fatigue life cycle result:

\begin{tabular}{|c|l|c|c|}
\hline No. & \multicolumn{1}{|c|}{ Description } & Result & Unit \\
\hline 1 & Addendum & 0.00125 & $\mathrm{~m}$ \\
\hline 2 & Dedendum & 0.001563 & $\mathrm{~m}$ \\
\hline 3 & $\mathrm{Rd}$ & 0.0459 & \\
\hline 4 & $\mathrm{Ra}$ & 0.0431 & \\
\hline 5 & Wt min & 2813.8 & $\mathrm{~N}$ \\
\hline 6 & Wt max & 2997.3 & $\mathrm{~N}$ \\
\hline 7 & Stress amplitude & 14.99 & $\mathrm{MPa}$ \\
\hline 8 & Mean stress & 474.87 & $\mathrm{MPa}$ \\
\hline 9 & $\mathrm{~B}$ & -0.0025 & \\
\hline 10 & $\mathrm{C}$ & 2.726 & \\
\hline 11 & $\mathrm{~N}$ (cycle) & $4.47 \times 10^{3}$ & \\
\hline
\end{tabular}

$$
\begin{gathered}
B=\left(-\frac{1}{3}\right) \times \log \left(\frac{0.8 S_{u t}}{S_{e}}\right) \\
C=\log \left[\left(0.8 S_{u t}\right)^{2} / S_{e}\right. \\
N=10^{-\frac{c}{b}} \times S_{a}^{\frac{1}{b}}=4.47 \times 10^{3} \text { cycles }
\end{gathered}
$$

\section{Conclusion and Recommendation}

Conclusion.

1. Based on the data that have been collected on chapter 3, the biggest bending stress happen on the ring gear which is $476 \mathrm{MPa}$.

2. From the data collected in the research of planetary gear on Toyota rush AT 2012, it can be assumed that the used material is equivalen to AISI 1050 that has yield strength of $515 \mathrm{MPa}$.

3. By using AISI 1050 material, the fatigue life cycle is categorized as low to high cycle fatigue $\left(\mathrm{N} \approx 10^{3}\right)$.

Recommendation.

1. Can be use as comparison for making or re-design planetary gear as mentioned using the material that has yield strength more than AISI 1050 (515 MPa), with safety factor that have been used can be bigger. Yet have to be count on cost on material and production.

2. Using material with properties above AISI 1050 that has UTS (Ultimate Tensile Strength) hight will got fatigue cycle that is higher or can be categorized as infinite life cycle. The fact that is happened on the field, very rarely happen but once it happen to be broken on the gear is caused of failure by age factor of the vehicle itself.

\section{References}

[1] Steven R.Schmid et al., Fundamental of Element Machine SI version third edition. New York: CRC Press, Taylor and Francis Group, 2014, pp. 392-393

[2] Steven R.Schmid et al., Fundamental of Element Machine SI version third edition. New York: CRC Press, Taylor and Francis Group, 2014, pp. 393-394

[3] Dr. Ala Hijazi. Meng 204. Lecture Notes, Topics: "Mechanical Drawing: Gears." The Hashemite University, Jordan. 2013 
[4] Robert L.Mott. Machine Elements in Mechanical Design" fourth edition. New Jersey: Pearson Education Inc, 2004, pp. 306-307

[5] Robert L.Mott. Machine Elements in Mechanical Design” fourth edition. New Jersey: Pearson Education Inc, 2004, pp. 330

[6] Robert L.Mott. Machine Elements in Mechanical Design" fourth edition. New Jersey: Pearson Education Inc, 2004, pp. 331-332

[7] Robert L.Mott. Machine Elements in Mechanical Design” fourth edition. New Jersey: Pearson Education Inc, 2004, pp. 333-334

[8] Dr. Ala Hijazi. Meng 204. Lecture Notes, Topics: "Mechanical Drawing: Gears." The Hashemite University, Jordan. 2013

[9] Dr. Ala Hijazi. Meng 204. Lecture Notes, Topics: "Mechanical Drawing: Gears." The Hashemite University, Jordan. 2013

[10] Robert L.Mott. Machine Elements in Mechanical Design" fourth edition. New Jersey: Pearson Education Inc, 2004, pp. 339-341

[11] Robert L.Mott. Machine Elements in Mechanical Design” fourth edition. New Jersey: Pearson Education Inc, 2004, pp. 309-310

[12] Dr. Ala Hijazi. Meng 204. Lecture Notes, Topics: "Mechanical Drawing: Gears." The Hashemite University, Jordan. 2013

[13] Dr. Ala Hijazi. Meng 204. Lecture Notes, Topics: "Mechanical Drawing: Gears." The Hashemite University, Jordan. 2013

[14] Robert L.Mott. Machine Elements in Mechanical Design" fourth edition. New Jersey: Pearson Education Inc, 2004, pp. 313

[15] Dr. Ala Hijazi. Meng 204. Lecture Notes, Topics: "Mechanical Drawing: Gears." The Hashemite University, Jordan. 2013

[16] Steven R.Schmid et al., Fundamental of Element Machine SI version third edition. New York: CRC Press, Taylor and Francis Group, 2014, pp. 383-386

[17] Dr. Ala Hijazi. Meng 204. Lecture Notes, Topics: "Mechanical Drawing: Gears." The Hashemite University, Jordan. 2013

[18] Steven R.Schmid et al., Fundamental of Element Machine SI version third edition. New York: CRC Press, Taylor and Francis Group, 2014, pp. 386

[19] Dr. Ala Hijazi. Meng 204. Lecture Notes, Topics: "Mechanical Drawing: Gears." The Hashemite University, Jordan. 2013

[20] Steven R.Schmid et al., Fundamental of Element Machine SI version third edition. New York: CRC Press, Taylor and Francis Group, 2014, pp. 384

[21] Dr. Ala Hijazi. Meng 204. Lecture Notes, Topics: "Mechanical Drawing: Gears." The Hashemite University, Jordan. 2013

[22] Dr. Ala Hijazi. Meng 204. Lecture Notes, Topics: "Mechanical Drawing: Gears." The Hashemite University, Jordan. 2013

[23] Dr. Ala Hijazi. Meng 204. Lecture Notes, Topics: "Mechanical Drawing: Gears." The Hashemite University, Jordan. 2013 
[24] Robert L.Mott. Machine Elements in Mechanical Design" fourth edition. New Jersey: Pearson Education Inc, 2004, pp. 483

[25] Robert L.Mott. Machine Elements in Mechanical Design" fourth edition. New Jersey: Pearson Education Inc, 2004, pp. 483

[26] Robert L.Mott. Machine Elements in Mechanical Design” fourth edition. New Jersey: Pearson Education Inc, 2004, pp. 367

[27] Steven R.Schmid et al., Fundamental of Element Machine SI version third edition. New York: CRC Press, Taylor and Francis Group, 2014, pp. 400

[28] Robert L.Mott. Machine Elements in Mechanical Design" fourth edition. New Jersey: Pearson Education Inc, 2004, pp. 372-373

[29] Robert L.Mott. Machine Elements in Mechanical Design” fourth edition. New Jersey: Pearson Education Inc, 2004, pp. 378-379

[30] Robert L.Mott. Machine Elements in Mechanical Design" fourth edition. New Jersey: Pearson Education Inc, 2004, pp. 387-388

[31] Robert L.Mott. Machine Elements in Mechanical Design" fourth edition. New Jersey: Pearson Education Inc, 2004, pp. 388-389

[32] Robert L.Mott. Machine Elements in Mechanical Design" fourth edition. New Jersey: Pearson Education Inc, 2004, pp. 389

[33] Steven R.Schmid et al., Fundamental of Element Machine SI version third edition. New York: CRC Press, Taylor and Francis Group, 2014, pp. 403

[34] Steven R.Schmid et al., Fundamental of Element Machine SI version third edition. New York: CRC Press, Taylor and Francis Group, 2014, pp. 403

[35] Steven R.Schmid et al., Fundamental of Element Machine SI version third edition. New York: CRC Press, Taylor and Francis Group, 2014, pp. 404

[36] Steven R.Schmid et al., Fundamental of Element Machine SI version third edition. New York: CRC Press, Taylor and Francis Group, 2014, pp. 404

[37] Robert L.Mott. Machine Elements in Mechanical Design” fourth edition. New Jersey: Pearson Education Inc, 2004, pp. 391-392

[38] ASM International. ASM Handbook Volume 19, Fatigue and Fracture. Materials Park, OH: ASM Intemational, 1996. 